\title{
A Testbed About Priority-Based Dynamic Connection Profiles in QoS Wireless Multimedia Networks
}

\author{
A. Toppan, P. Toppan, C. De Castro and O. Andrisano \\ IEIIT-CNR, National Research Council of Italy \& WiLab, \\ University of Bologna, Bologna, \\ Italy
}

\section{Introduction}

The ever-growing demand of high-quality broadband connectivity in mobile scenarios, as well as the Digital Divide discrimination, are boosting the development of more and more efficient wireless technologies.

Despite their adaptability and relative small installation costs, wireless networks still lack a full bandwidth availability and are also subject to interference problems.

In context of a Metropolitan Area Network serving a large number of users, a bandwidth increase can turn out to be neither feasible nor justified. In consequence, and in order to meet the needs of multimedia applications, bandwidth optimization techniques were designed and developed, such as Traffic Shaping [1-3], Policy-Based Traffic Management [48] and Quality of Service (QoS) [9-17].

In this paper, QoS protocols are adopted and, in particular, priority-based dynamic profiles in a QoS wireless multimedia network. This technique [18-20] allows to asssign different priorities to distinct applications, so as to rearrange service quality in a dynamic way $[21,22]$ and guarantee the desired performance to a given data flow.

In particular, the platform can manage two levels of priority: among different users and within a single user's connection.

In the former kind of priority management, those users whose guaranteed bandwidth is higher, will be proportionally assigned a greater part of the shared bandwidth.

The latter case refers to each single user, whose distinct services are assigned distinct priorities. Each profile, in fact, allows the real time management of services, and the priority parameter is used to queue the desidered services properly.

A complete testbed involving 80 users approximately is here presented, where such technique is adapted to the specific requirements of the plant.

The network infrastructure installation is detailed, the whole QoS system developed is described and four measurement campaigns are reported. 
The whole testing was directed by WiLab (www.wilab.org), which includes the IEIIT-CNR (National Research Council of Italy, IEIIT Bologna unit) and a portion of the TLC scientific community at Bologna University (Italy). The design and technical aspects of the problem were and are still being carried out by such group.

The proposed platform aims at supporting a Wireless Internet Service Provider (WISP) in the management of its network infrastructure in a user-friendly and straightforward way. It can be accessed through the Internet and lets the network manager define different access profiles and supervise all the users' connections.

The QoS service, in particular, allows to set each user's minimum bit rate guaranteed and maximum supported, enable services such as VoIP, FTP, Mail and P2P and assign them specific priorities.

The network scenario installed and used for the testbed includes an Internet gateway, a server which hosts the whole infrastructure control system and five sectoral distribution devices.

The software platform allows to define some distinct kinds of priority-based connection profiles, each characterized by a set of different parameters and a diverse commercial value. Personal data can also be managed, each corresponding to the user's kind of connection profile subscribed.

Reports about connections and traffic statistics are also at disposal, also useful to law purposes. A continuous monitoring of the wireless network infrastucture is also possible.

All the above features can be easily managed through specific user-friendly portals.

This kind of services are fundamental in many application fields, ranging from Intelligent Transportation Systems (ITS) and Infomobility to "Smart Cities", where wireless applications guide the user in most of his activities.

The paper is organized as follows: Section 2 describes the testbed setup and the QoS software developed. Section 3 details the results of the four measurements campaigns.

These techniques, addressed to to real applications, are discussed in the following: the PEGASUS project about the support of real time in Infomobility is discussed in Section 4. The Smart Cities scenario is presented in Section 5. Conclusions and future tesbed extensions are discussed in Section 6.

\section{The network infrastructure and QoS system}

Although the main purpose of this work is to present measurements, it is important to describe the testbed setup and some related installation problems, as well as the QoS system and its main principles.

\subsection{Testbed setup}

The network plant is depicted in Fig. 1 and, from the left to the right, involves a Shelter for Internet distribution (A) which, due to property reasons, could not host the QoS Management Server (B), installed $1.2 \mathrm{~km}$ away (Link 1). 
Such system runs on a Dual Xeon $2 \mathrm{GHz}$, 8Gb di Ram, 80Gb SAS Raid 5 server and comprehends a Radius authentication server, a PPPoE concentrator and the whole QoS management software.

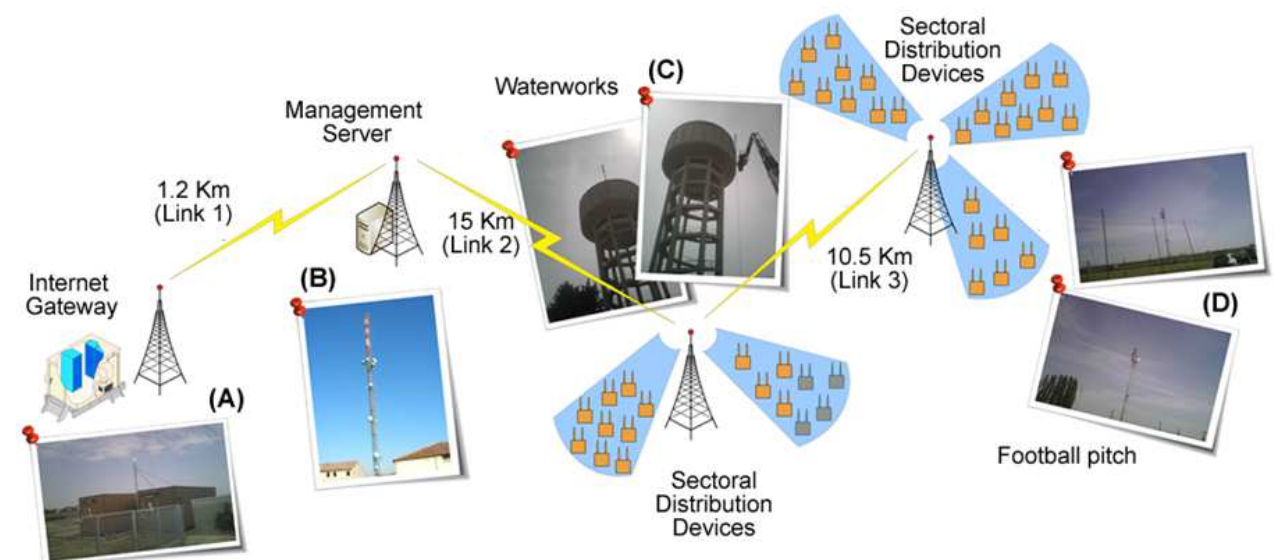

Fig. 1. the QoS equipment.

A HiperLAN link starts here and connects the management server to a waterworks area (C) $15 \mathrm{~km}$ away (Link 2). In such area, two sectoral distribution equipments were setup and serve 35 users approximately.

A further antenna allows to reach the last distribution area, situated near a football pitch (D) $10.5 \mathrm{~km}$ away (Link 3), and including three further sectoral distribution equipments for 45 users approximately. Such antenna had to be setup since some unfavourable features of the ground prevented a direct connection from being created.

The indirect link creates a bottleneck and forces the waterworks area to support some of the traffic surrounding the pitch.

A further penalty is that the same pylon which hosts the antenna in area (C) also carries some television and microwave aerials. In consequence, some devices not optimally shielded were initially blocked and even damaged and the available bit-rate is still being diminished.

In addition, some further installation problems were caused by the daily activation of the waterworks pump, which produced strong perturbations to the electric network, consequent blocks of many devices and even breakdowns. This problem was solved by means of an electronic filter.

All the above problems would have obviously prevented 80 users from being propely served, unless a bandwidth optimization method and traffic management were adopted.

\subsection{The QoS architecture adopted}

The QoS scheme is based on the dynamic assignment and redistribution of bandwidth on the basis of priority and users' profiles. The main parameters of each kind of profile are 
summed up in Tab. 1. In particular, when QoS management is enabled in a profile (QoS flag), different priorities can be assigned to distinct protocols.

\begin{tabular}{|l|l|}
\hline \multicolumn{1}{|c|}{ Parameter } & \multicolumn{1}{c|}{ Description } \\
\hline Name & Profile name \\
\hline Description & Profile description \\
\hline Upload bandwidth & Max upload bandwidth (kbit/s) \\
\hline Upload guaranteed & Min upload bandwidth guaranteed (kbit/s) \\
\hline Download bandwidth & Max download bandwidth $(\mathrm{kbit} / \mathrm{s})$ \\
\hline Download guaranteed & Min download bandwidth guaranteed (kbit/s) \\
\hline QoS & Flag for enabling QoS traffic management \\
\hline
\end{tabular}

Table 1. main fields of a connection profile.

Fig. 2 shows the graphic interface for the definition and management of each type of profile. The seven panels "Band 1",... "Band 7" on the right allow to assign priorities, 1 being the highest, 7 the lowest. In particular, a protocol can be associated to a specif priority by dragging and dropping its name in the chosen band panel.

The minimum bandwidth guaranteed (in percentage) and maximum available must also be setup for each sub-bandwidth. Once a profile has been defined, it can be assigned to many distinct users, so as to tailor service supply easily and quickly.

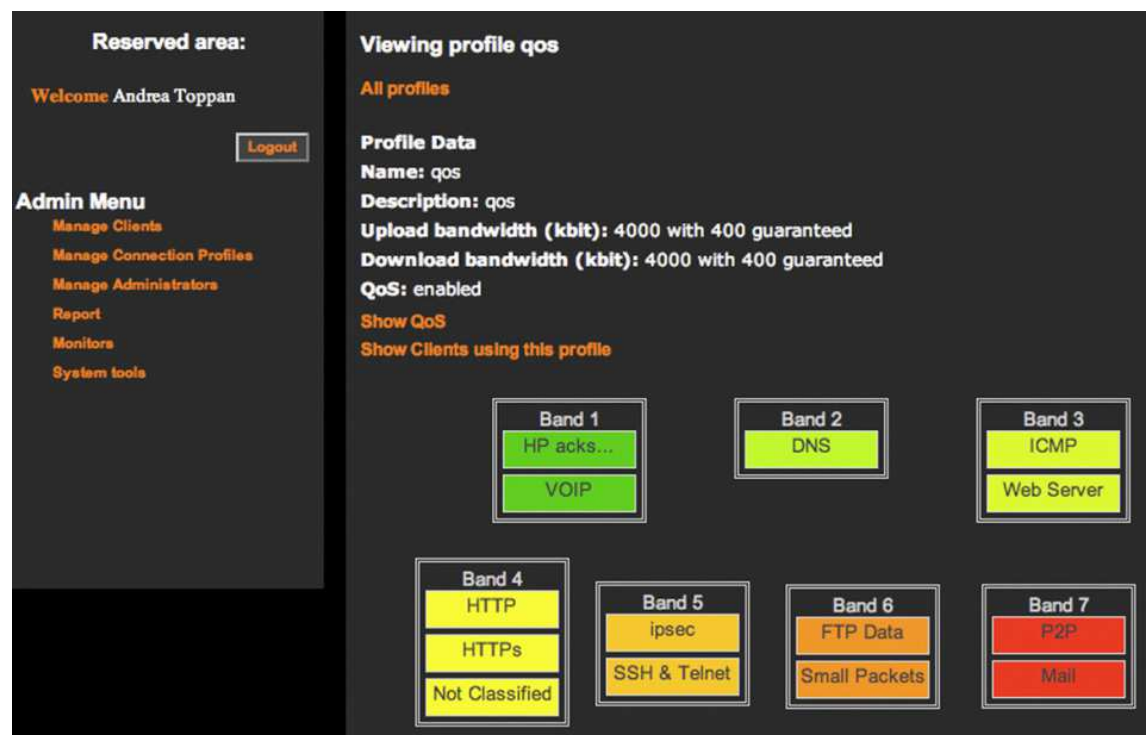

Fig. 2. priority assignment through drag\&drop operations.

As far as dynamic QoS management is concerned, the basic idea is to limit both upload and download operations through the Egress policier (www.egress.com), so as to discard 
all the packets whose speed exceeds a maximum value set. To this purpose, queuing algorithms are applied and bandwidth can consequently be tuned according to actual availability.

Some problems had to be solved along the way: shaping could only be applied to the outgoing traffic, already processed by the kernel, whereas both the uploading and downloading flows should normally be shaped by queuing methods on both the incoming and outcoming interfaces. In this case, though, many independent PPP interfaces were simultaneously active and each PPPoE was thus identified through a PP system interface numbered $\mathrm{N}(\mathrm{PPN}, \mathrm{N}=0,1, \ldots)$.

In consequence, two further difficulties had to be faced:

1. too many iptables rules were generated and so was a further branch in the queuing structures;

2. the htp qdisc bandwidth sharing capabilities could not be fully exploited and no minimal bandwidth per PPP connection could be guaranteed.

As a matter of fact, each PPP having its own independent queuing, the traffic on the network interface was managed in an unpredictable way: no minimum bandwidth per connection could be even assigned and the unexploited bandwidth could not be dynamically and equally redistributed among all connections.

In order to handle the above situation, a qdisc (common to all connections) and subclasses for each kind of connection (with minimum and maximum bandwidth set) were defined, so as to redistribute unexploited resources among tunnels.

To this purpose, a hierarchical structure based on HTB (Hierarchical Token Bucket, http://luxik.cdi.cz/ devik/qos/htb) queuing was developed, whose nodes specify their own minimum and maximum bandwidth. In this way, the traffic of each tunnel is forwarded to the class it pertains to, so as to achieve the desired result.

Nevertheless, qdiscs can only manage the traffic of their own interface, so it was still impossible to identify a single connection by accessing the network interface of the PPPoE server. Each connection, in fact, is managed as a separate network interface.

An IMQ (Intermediate Queueing Device, www.linuximq.net) interface was thus adopted, which allows to manage qdiscs and the whole traffic: iptables are deviated to such interface and traffic can be shaped. Each single PPP interface must be assigned a connection identifier and sent to the IMQ, where connection classes were defined.

In this way, each connection can monitored, traffic can be classified on the basis of protocols and the most important flows are assigned the highest priority.

Another problem faced was that each packet could only be marked by means of an an identifier, so, theoretically speaking, the simultaneous identification of connections and protocols within a session was impossible.

Several tests demonstrated that the problem could be solved through the joint use of u32filter+MARK and CLASSIFY TARGET. This was done defining a further HTB class structure in the PPPN interfaces. 
The bandwidth redistribution problem having been solved, attention could be focused on flow priority within each connection.

Fig. 3 shows how queuing algorithms were applied. A root node (qdisc) was created in the imq0 interface; class 1:1 was added in order to define the total bandwidth $(100 \mathrm{Mbit} / \mathrm{s}$ in this case). Subclasses were then defined for the management of single connections, each specifying the minimum guaranteed (rate) and maximum at disposal (ceil).

Note that a higher QoS could be achieved if the SFQ (Stochastic Fair Queuing) were applied, so as to manage single flows through a Round Robin policy.

In order to manage priority of single flows, a hierarchic structure was created within each PPPN interface.

As in the former case, several subclasses were added to the root node (qdisc), each with a minimum and maximum bandwidth; the SFQ algorithm was applied afterwards. A u32filter list is defined in the root node of each interface, so as to drive single packets on the class they pertain to on the basis of their protocol. Packets were initially divided by the CLASSIFY TARGET tool according to the connection; a further division was then made by u32filter+MARK on the basis of protocols.

Besides, the identifiers range is 1:10000 and 1:65535 in hex, so the highest attention must be paid to each class handles. The following synatx was adopted:

$$
\text { iptables -t mangle -A POSTROUTING -j CLASSIFY --set-class x:y }
$$

In this way, filters could be avoided and everything is managed by iptables.

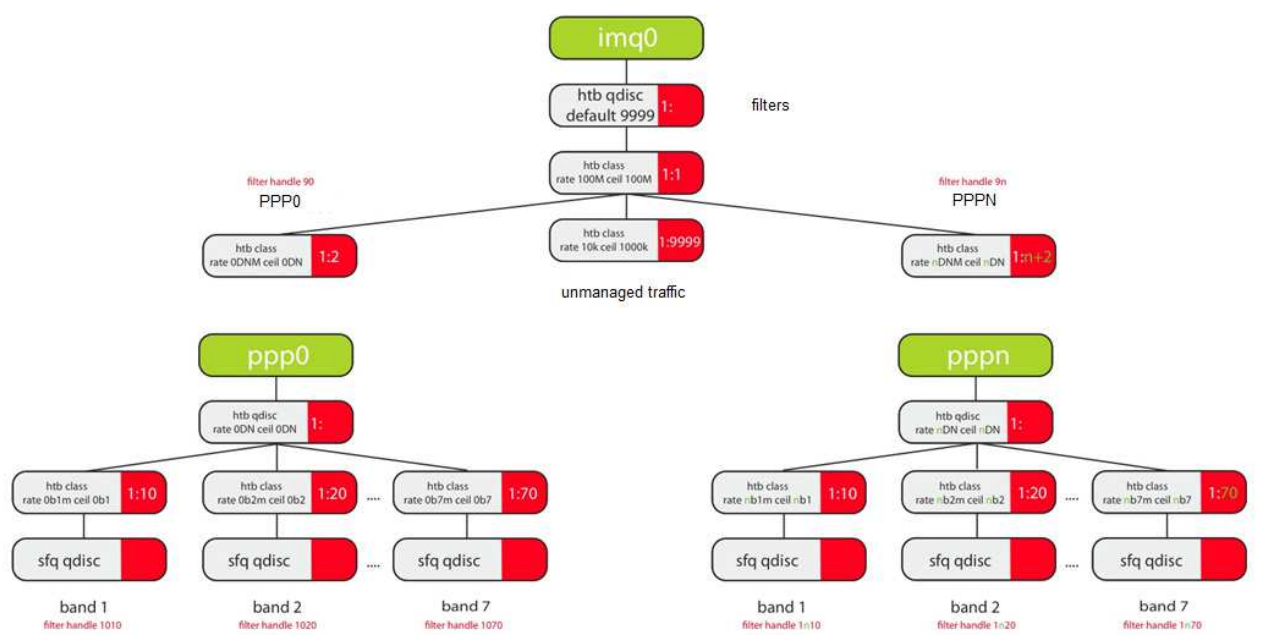

Fig. 3. hierarchical QoS management. 


\subsubsection{Statistics}

The platform allows to visualize information about the infrastructure and its use and, in consequence, to make statistics about connected users and their traffic volume.

Fig. 4, for instance, refers to all the users' total connection time. For the sake of compactness, only a small excerpt was reported.

Diagrams are also available describing the system components, such as CPU load, network load and many others.

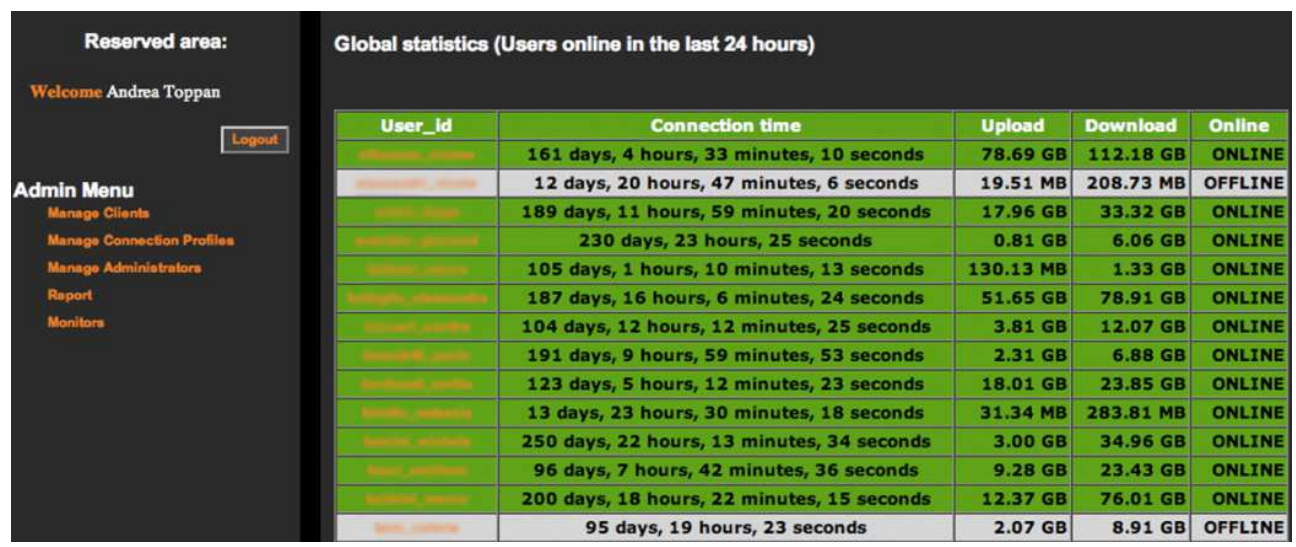

Fig. 4. small excerpt from all the users' total connection time.

As far as each user is concerned (Fig. 5), the following data can be monitored: connections, data volumes exchange, diagrams about his or her traffic and, as indicated by law, packets logging.The authentication system adopted is Radius and traffic is encapsulated through the PPPoE protocol. In consequence, a PPP tunnel is active between each user and the server.

\section{Measurements campaigns}

As already anticipated, the main concern of this paper is to present a realistic testbed for QoS management; four measurements campaigns carried out are described in the following, whose scenario was described in Section 2.1 (Fig. 1).

The adopted dynamic priority-based method is twofold. On the one hand, users are assigned the shared bandwidth on the basis of their profiles: the higher the guaranteed bandwidth, the higher the shared bandwidth assigned. On the other hand, not only users but also services within a profile are prioritized, so each user is aware that his or her bandwidth is accordingly shared among his or her applications.

The QoS management is presented in an increased way: no QoS in the first campaign; QoS with neither minimum nor maximum set in the second; minimum and maximum bandwidth defined in the third; dynamic redistribution is also managed in the fourth. 


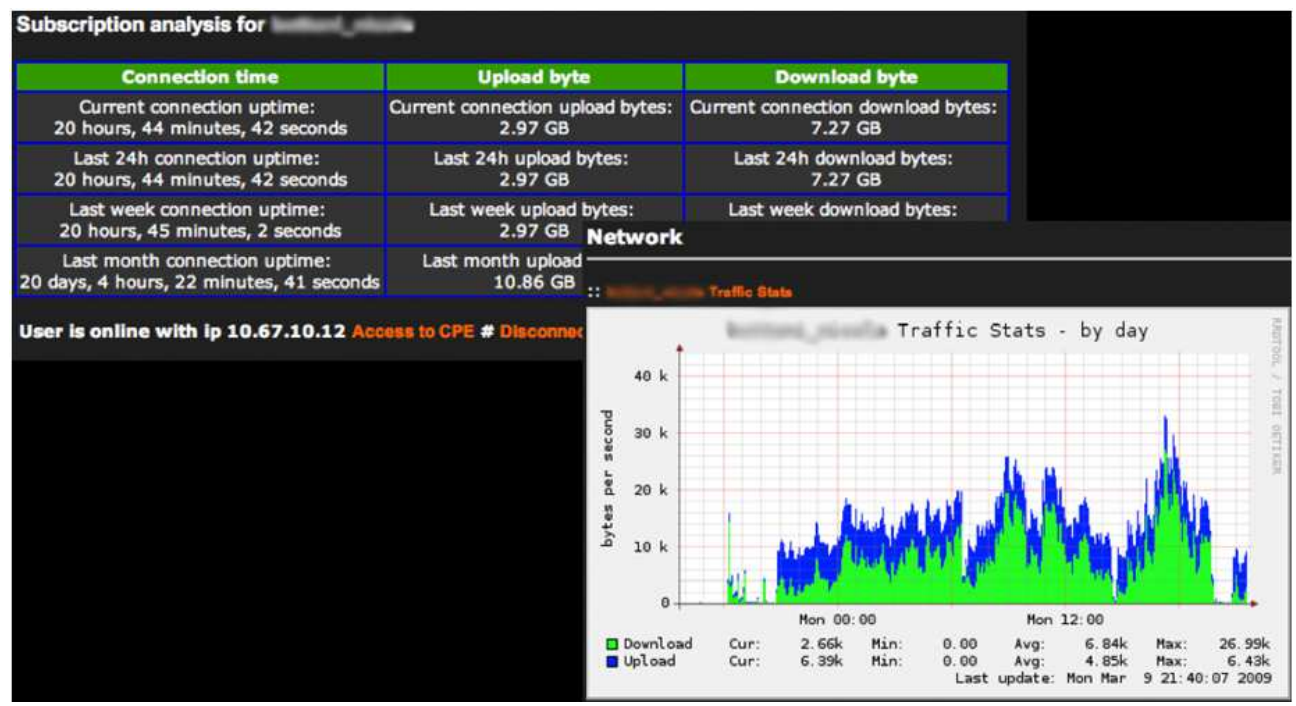

Fig. 5. statistics about a single user.

\subsection{Throughput on single links (no QoS)}

The first campaign represents throughput maximization in the single links of the whole infrastructure. Fig. 6 shows results in Link 1, from the Shelter to the Management Server.

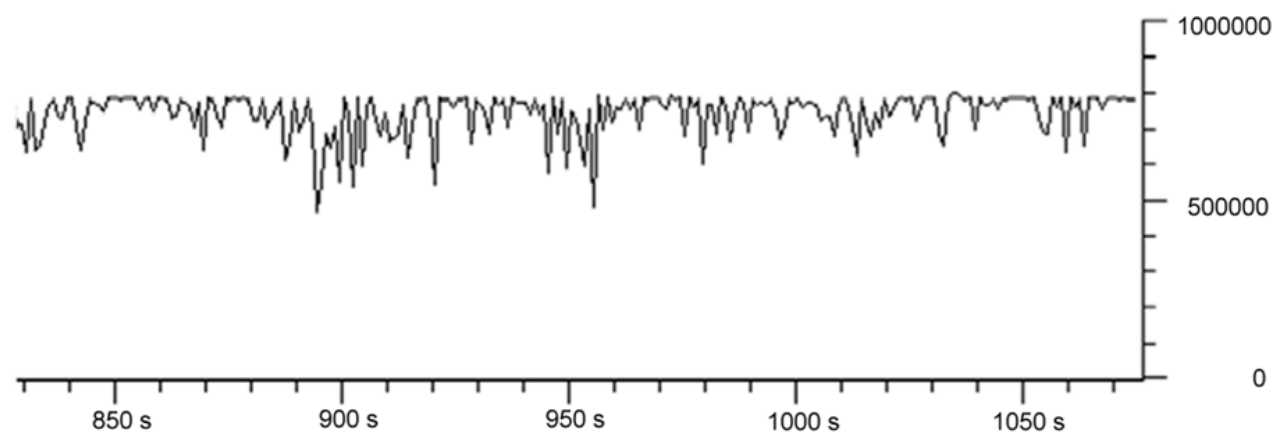

Fig. 6. throughput in the link from the Shelter to the Management Server (bit/s).

The above measurements were performed using the following tools: (1) Iperf (www.noc.ucf.edu/Tools/Iperf), which allows to send a TCP or UDP data streams and measure their throughput; (2) WireShark (www.wireshark.org), a network analyzer which allows to capture and diagram Iperf streams.

In this campaign, the Iperf server was installed on an Acer Travelmate with Linux Debian OS and located in the B node, so as to receive UDP connections. A Macbook Pro with Mac OSX Leopard was used as the client. 
Nodes A, C, D hosted laptops for the connection to the Iperf server. In this way, throughput could be measured first in Link1, then Link2 and Link 3 and finally in Link2 + Link3.

The highest UDP throughput at disposal in Iperf connnections was set to $10 \mathrm{Mbit} / \mathrm{s}$; results are reported in Tab. 2 and derive from a large number of measurements properly mediated.

Note that the throughput from the Management Server to the football pitch (Link $2+$ Link 3) is almost 2Mbit/s lower than in single links 2 and 3: this derives from the the same device being charged of both signal reception and transmission.

\begin{tabular}{|l|l|l|}
\hline \multicolumn{1}{|c|}{ Link } & \multicolumn{1}{c|}{ Description } & \multicolumn{1}{c|}{ Throughput } \\
\hline Link 1 & From the Shelter to the Management Server & $7.8 \mathrm{Mbit} / \mathrm{s}$ \\
\hline Link 2 & Management Server to Waterworks & $6.6 \mathrm{Mbit} / \mathrm{s}$ \\
\hline Link 3 & Waterworks to Football pitch & $6.9 \mathrm{Mbit} / \mathrm{s}$ \\
\hline Link2 + Link 3 & Management Server to Football pitch & $5.2 \mathrm{Mbit} / \mathrm{s}$ \\
\hline
\end{tabular}

Table 2. results in single links.

\subsection{QoS applied to multimedia TCP flows (no $\mathrm{min} / \mathrm{max}$ bandwidth set)}

The second campaign aimed at verifying the efficiency of the QoS management server. The Iperf was moved in the $(\mathrm{C})$ node, so as to check Link 3.

An ethernet cable subsituted the wireless connection during a PPP connection. Delays and packet loss, in fact, are not particularly relevant in this kind of control, attention being mainly focused on flow management.

The following tools were adopted: (1) QoS Server; (2) Server-side Vlc for MMS over http video flow transmission (www.videolan.org/vlc); (3) Client-side Vlc for flow reception; (4) WireShark.

Fig. 7, diagrammed through WireShark, represents the scenario and the first measurements of this campaign. It refers to the following profile: no QoS applied, symmetric upload and dowmlod of $1 \mathrm{Mbit} / \mathrm{s}$, no bandwidth guaranteed. The client receives the first video (red line) until second 230, then the second video (green line) starts and the firts one is interrupted at second 280 .

As expected, in the concurrent period (sec. 230 to 280) both TCP videos are blocked, the bandwidth being inadequate to support both of them.

Fig. 8 represents the second test and involves three videos on three distinct ports; in this case, a QoS profile was enabled which guarantees an increasing priority from the first to the third flow.

First starts the video on the lowest priority port (blue curve); the intermediate priority video starts 20 seconds later (green curve) and, in consequence, the first data flow declines. In the period between sec. 40 to 80 the maximum throughput was increased, so as to emphasize the effect of dark and still scenes in the second video. In this way, the total throughput is constant and bandwidth waste is kept under control. 


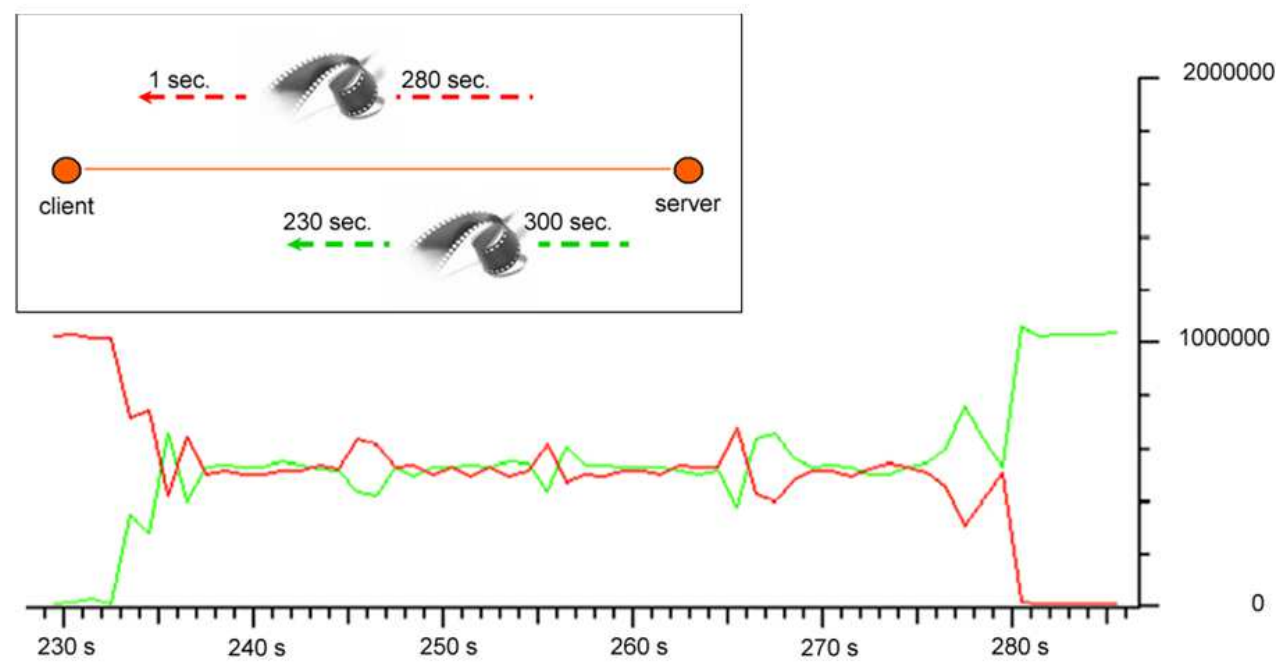

Fig. 7. two concurrent video flows without QoS (bit/s): scenario and results.

The same observations apply to the third and highest priority flow (red curve): the three videos share the bandwidth and, thanks to QoS, the lowest priority one is flattened, the medium is assigned less bandwidth and the highest has the best quality.
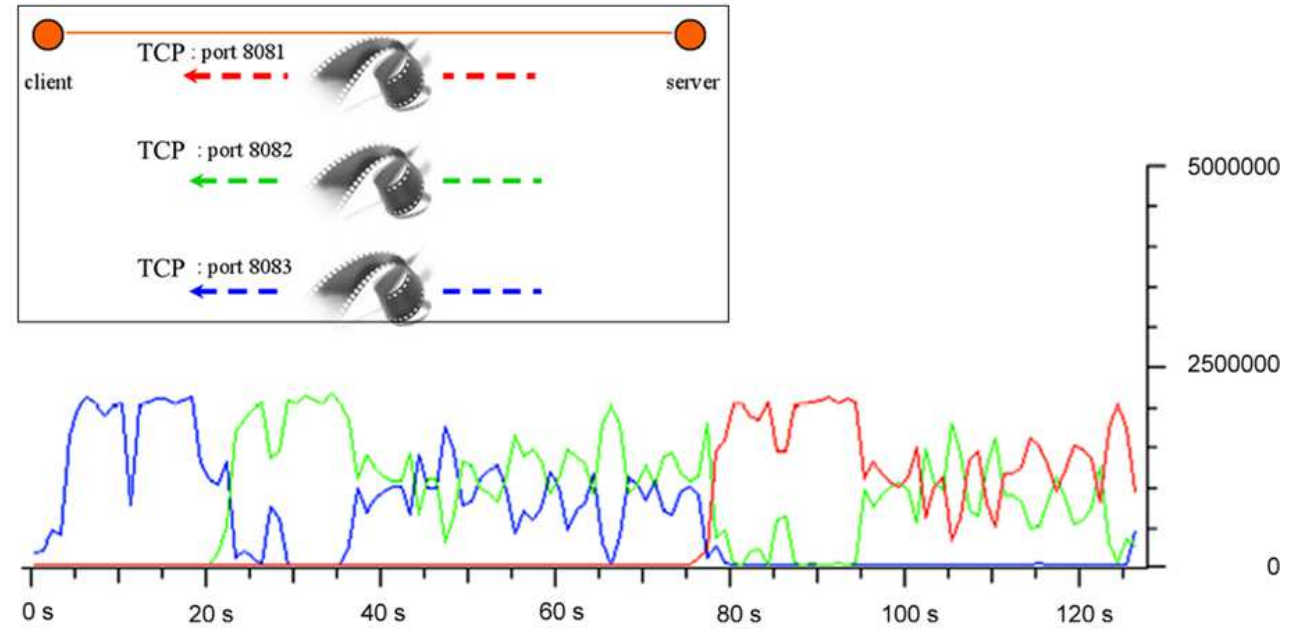

Fig. 8. three concurrent videos with QoS (bit/s).

\subsection{Bandwidth control in QoS management}

As the previous measurements showed, the QoS policy adopted helps to avoid bandwidth waste and guarantees a better service, especially for VoIP, IPTV support etc. 
Nevertheless, this kind of priority management among traffic classes implies the almost complete cancellation of the less important services for the benefit of the most important ones.

In the initial QoS profile, an increasing priority was assigned to the three TCP flows on distinct ports. Fig. 9 shows the measures obtained. The less priority flow (blue curve) is strongly limited by the second one (green curve). They are both flattened when the highest priority flow starts (red curve).

In order to improve such results, each traffic class was then assigned a minimum and a maximum throughput (Tab. 3). The third campaign tries to demonstrate the effectiveness of such method and Fig. 10 reports the results.

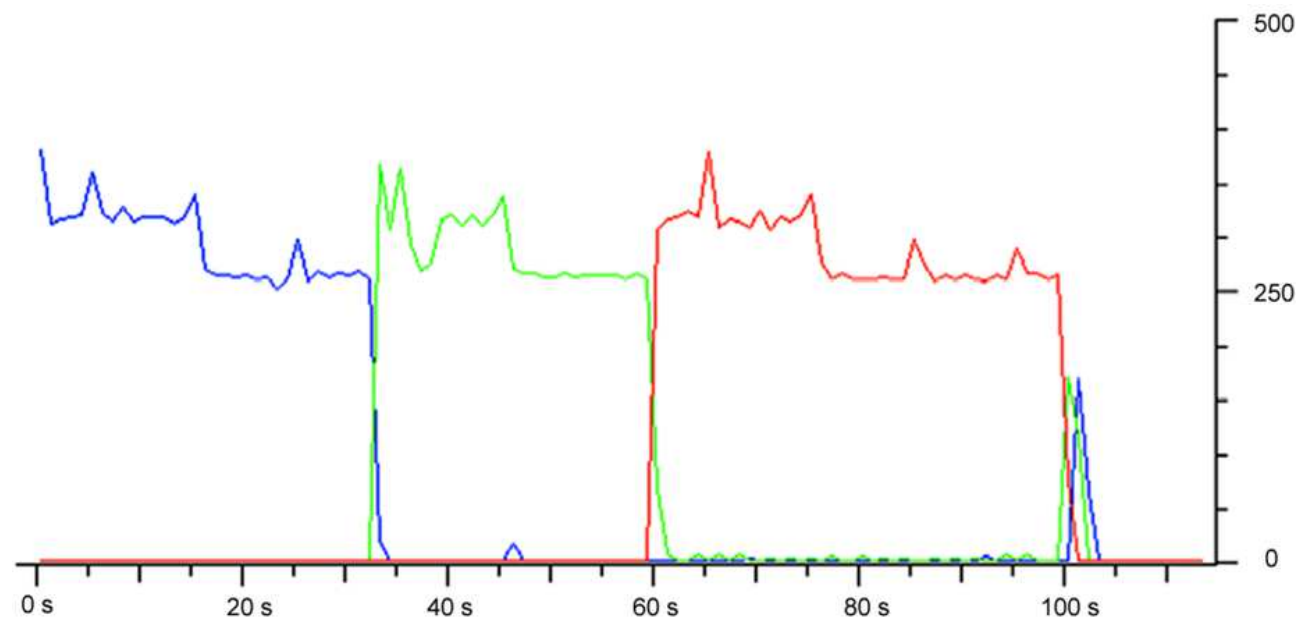

Fig. 9. TCP concurrent flows with QoS (bit/s).

\begin{tabular}{|l|l|l|l|}
\hline Class & \multicolumn{1}{|c|}{ Priority level } & \multicolumn{1}{|c|}{$\begin{array}{c}\text { Min. bandwidth \% } \\
\text { guaranteed }\end{array}$} & \multicolumn{1}{|c|}{$\begin{array}{c}\text { Max. bandwidth \% at } \\
\text { disposal }\end{array}$} \\
\hline 1 & Highest priority & $0 \%$ & $100 \%$ \\
\hline 2 & Intermediate & $30 \%$ & $30 \%$ \\
\hline 3 & Lowest priority & $20 \%$ & $100 \%$ \\
\hline
\end{tabular}

Table 3. minimum throughput guaranteed and maximum available, as assigned to single flows.

Note that an upper bound having been imposed to the intermediate flow, the lowest is not totally flattened, but only slowed down.

The most priority flow starts at second 50 and, in consequence, the less priority traffic becomes slower, but not more than the $20 \%$ guaranteed. The same applies to the intermediate flow, for which a $30 \%$ at least is available. The highest priority flow, of course, can not reach the maximum speed. 
As this measure shows, if guaranteed bandwidth percentages are properly managed, a high QoS can be obtained in an easy and immediate way.

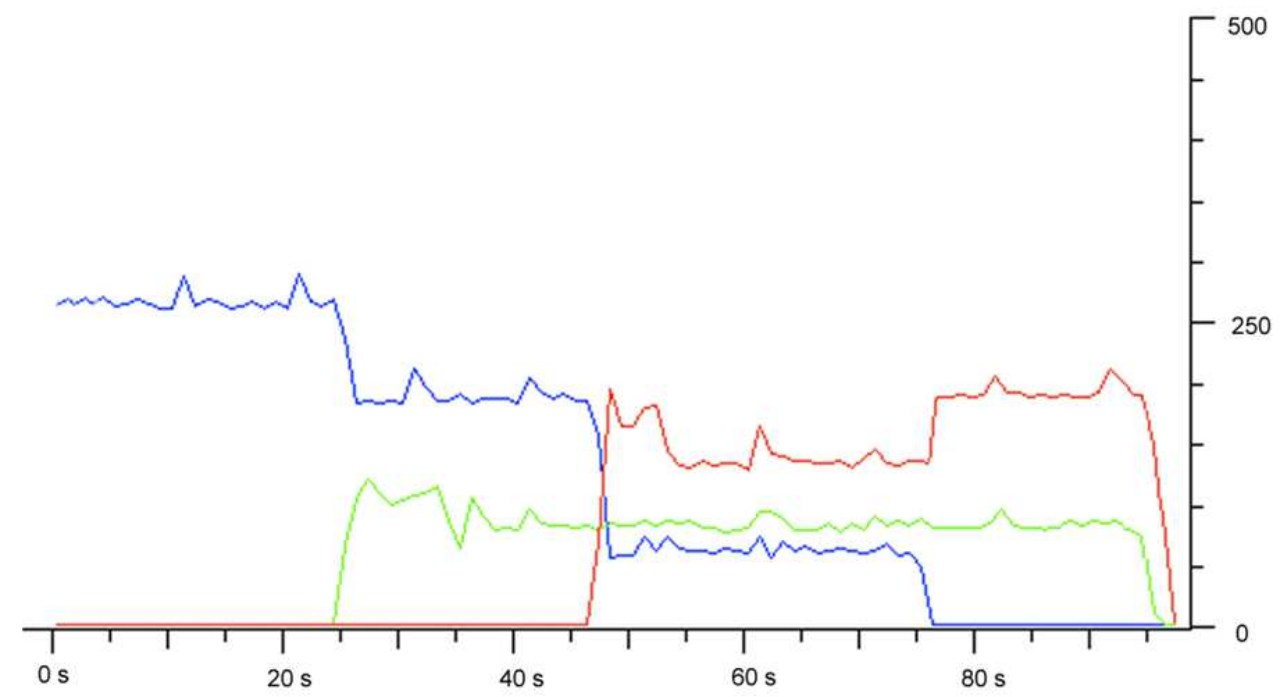

Fig. 10. TCP concurrent flows with bounded bandwidth QoS (bit/s).

\subsection{Proportional reassignment of bandwidth}

An important feature that must be handled in this kind of QoS management is bandwidth reassignment proportionally to each user's minimum guaranteed.

In this case, three PCs and the usual tools were adopted and two kinds of profiles were defined (Tab. 4).

\begin{tabular}{|l|l|l|}
\hline \multicolumn{1}{|c|}{ Clients } & \multicolumn{1}{c|}{ Min. guaranteed } & \multicolumn{1}{c|}{ Max. at disposal } \\
\hline Clients 1 and 2 & $128 \mathrm{Kbit} / \mathrm{s}$ & $3 \mathrm{Mbit} / \mathrm{s}$ \\
\hline Client 3 & $600 \mathrm{Kbit} / \mathrm{s}$ & $3 \mathrm{Mbit} / \mathrm{s}$ \\
\hline
\end{tabular}

Table 4. Minimum and maximum throughput for each client.

Clients were connected to the server through the PPPoE protocol; the maximum throughput between clients and server was set to $3 \mathrm{Mbit} / \mathrm{s}$, so as to simulate a set of wireless relays.

In this case, the upper bandwidth was to be shared among concurrent users and, in consequence, none was to reach the maximum.

Results are shown in Fig. 11: initially, the only connected client 1 (green curve) gets the whole bandwidth available according to his profile (3 Mbit/s).

After 100 seconds approximately, client 2 is also connected, throughput is assigned according to the minimum guaranteed and exceeding bandwitdh is reassigned according to such value. 
The two clients share the same profile, so the bandwidth is equally divided and redistributed.

At second 150 the third client (red curve) connects to the system; the minimum throughput of his or her profile is four times the others', so clients 1 and 2 are limited accordingly.

When client 1 disconnects, bandwidth is distributed among clients 2 and 3 in a ratio of 1 to 4 .

Finally, client 3 logs out and the whole bandwidth is at client's 2 disposal.

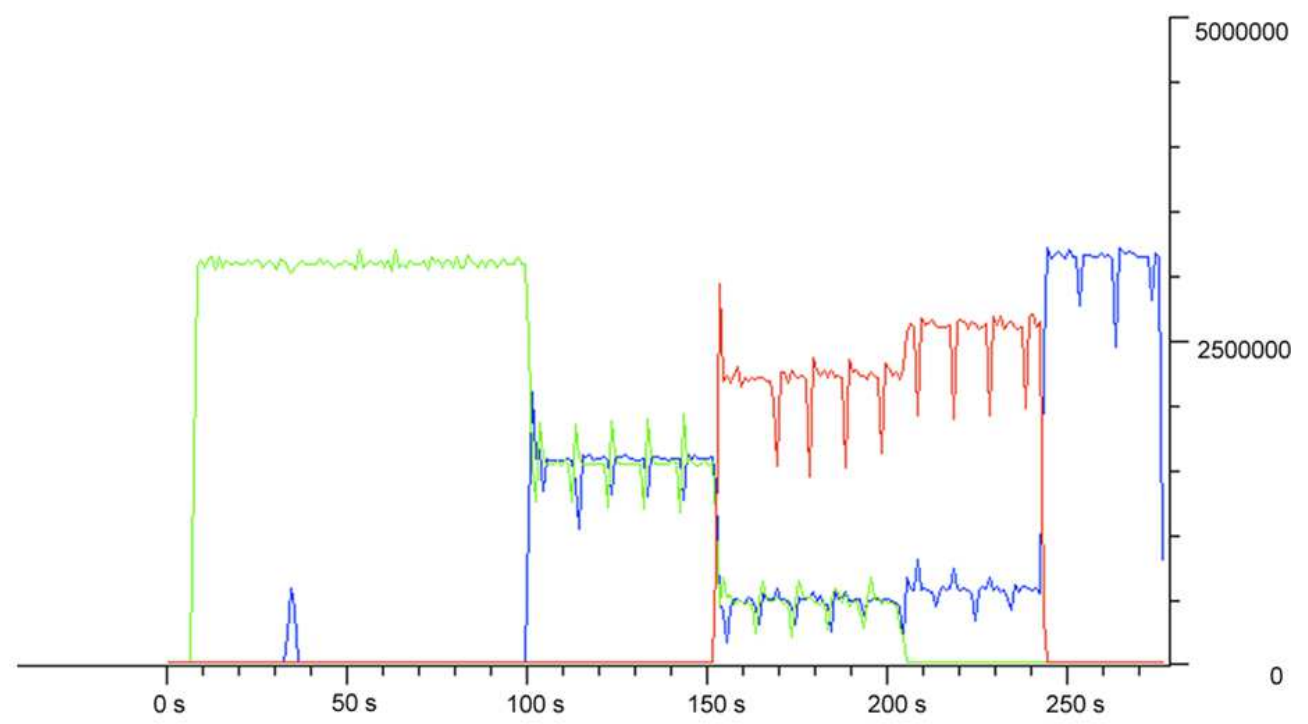

Fig. 11. bandwidth proportional reassignment among connected users.

\section{The PEGASUS project: Real time support in infomobility services}

One of the most important applications to which QoS techniques have been applied are Smart Navigation and the braoader field of Infomobility.

Transportation is one of the main fields where advanced technological systems can improve human life in a significant way: risks due to accidents, time wasted travelling and pollution could be highly reduced by applications for vehicle localization, behaviour prediction, etc.

These considerations are at the basis of the increasing interest that ITS are gaining in these years.

Furthermore, the latest study on global urbanization conducted by the Population Division of the Department of Economic and Social Affairs of the United Nations predicts that, in 2050 , nearly $70 \%$ of the global population will be living in larger cities [23].

This immense aggregation of people will surely pose great challenges to the sustainability of modern lifestyle, and the problem of an efficient management of mobility stands out as one of the most relevant ones. 
As a matter of fact, densely populated cities imply the concentration (from the country) and distribution (within the city) of massive amounts of people and resources [24].

In addition to the vast economic importance and consequences of such situation, urban and sub-urban mobility is a serious challenge also due to the circulation of large amounts of people and goods in a relatively small area. This poses hazards to life and health, especially for children, the elderly, and unfamiliar visitors, as well as to the environment.

Urban mobility, in fact, accounts for some $30 \%$ of energy consumption and $70 \%$ of transport pollution in Europe, and this problem is magnified by the increasing population concentration in large cities.

In such a scenario, the efficient management of traffic is a challenge that governments, industries and researchers are forced to face worldwide. Private travellers, commercial road users, and the public sector are continually searching for new and faster travel routes and methods.

Roads efficiency can be substantially improved by the deployment of ITS, which exploit ICT in order to provide traffic safety and efficiency.

ICT can be considered as the foundation for carrying out smart navigation, meant as the paradigm where mobile entities (vehicles and pedestrians) move wisely through a given environment, exploiting reliable and timely information about traffic conditions.

In this context, one of the most important applications is the support of real time, meant as the constant monitoring of traffic and road conditions, and the consequent possible update of the routes previously suggested. As a matter of fact, the best path in a given situation can vary when traffic conditions vary and updates should be notified to the user in real time. Nevertheless, up to now, no simple and marketable product was proposed for monitoring traffic and providing real time information to road users.

To this purpose, in the framework of the Italian project PEGASUS (http://pegasus.octotelematics.com/), WiLab aims at exploiting information transmitted from vehicles to a remote Control Center, so as to provide drivers in real time with updated information about actual traffic conditions. In this way, a new smart navigation service is supported. In particular, the objective is twofold:

- investigate the impact of smart navigation on the communication networks load;

- investigate the impact of real time updates on traffic management efficiency; as a matter of fact, vehicles equipped with smart navigators are constantly sent information about actual roads conditions;

In Fig. 12, the smart navigation scenario considered and developed at WiLab is shown: vehicles are equipped with on-board units (OBUs), which periodically transmit their speed and position (known through the GPS integrated on board) to a Control Center. Such data are transferred through the General Packet Radio Service (GPRS) network.

The fleet equipped with OBUs is addressed as floating car data (FCD). In March 2010, the Italian FCD to which the PEGASUS project refers, reached over 1.000 .000 equipped vehicles (OctoTelematics, 2010); this number is to increase quickly (note that the number of public 
and private vehicles in Italy was 34 million back to 2003 [25], hence the FCD is a not negligible percentage of the overall private vehicles number).

All such data are processed and exploited for the real time dynamic navigation of vehicles (hereafter Dynamic Route Guidance, DRG); the same information can also be forwarded to public or private institutions for traffic management, etc.

In the near future, almost all vehicles will be able to send real time information, and the majority of drivers will take profit of data properly processed and of applications beyond traffic management, such as safety and entertainment.

In this scenario, telecommunications systems will be required to transmit information quickly and reliably, both among vehicles and between vehicles and remote control centers. Which technologies are to be chosen, how priorities must be managed, which capacity is required, are still open issues.

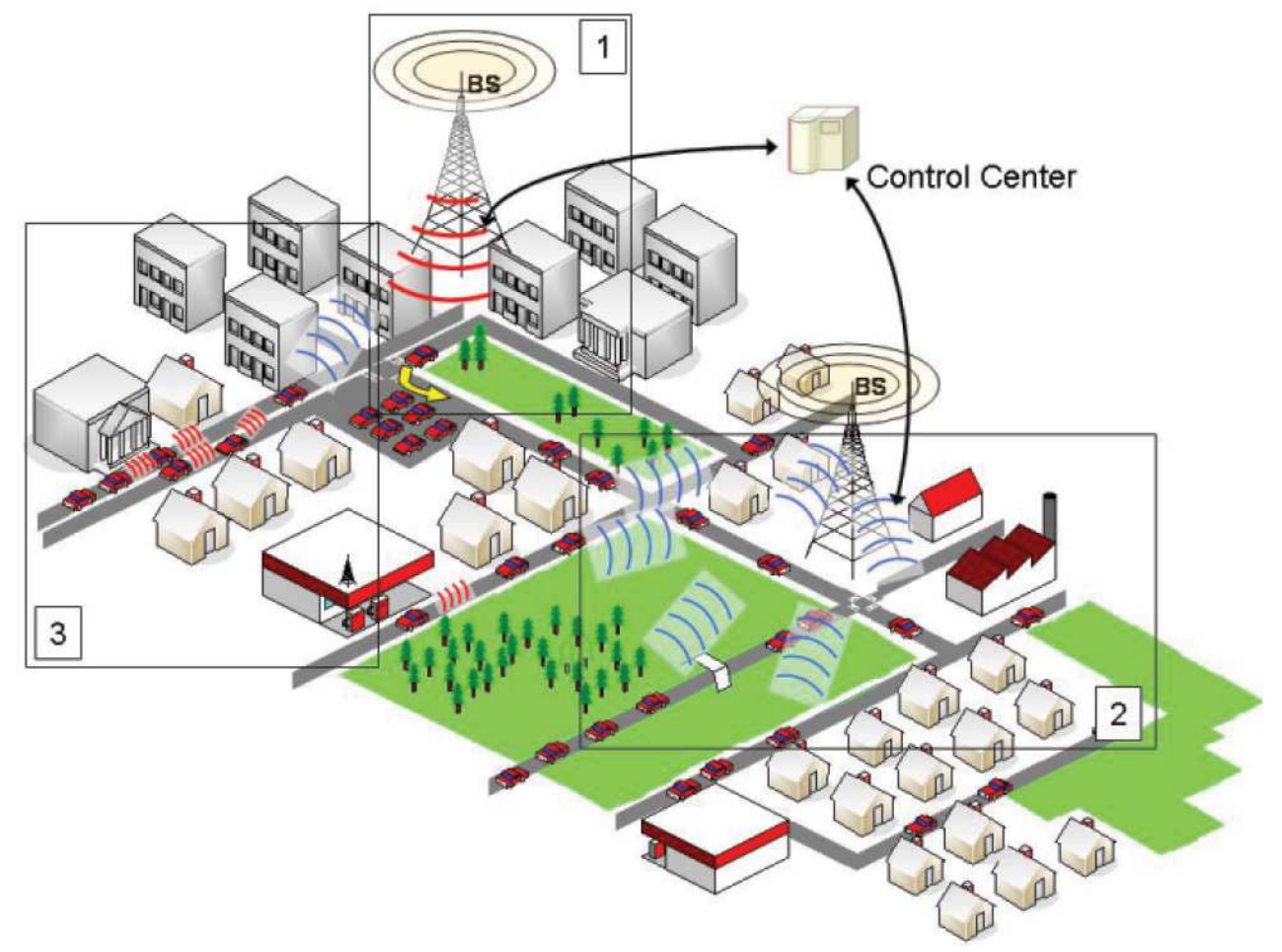

Fig. 12. Smart navigation scenario.

The mobile network is, at present, the only one adopted for vehicles-Control Center communication; nevertheless, the quantity and size of information is to increase.

Urban networks, thus, can turn out to be a precious support for existing infrastructures, especially if properly managed through effective QoS techniques. 


\section{The smart cities scenario}

The QoS testbed is currently being applied to Smart Cities scenarios, a class of applications which are gaining an increasing attention.

As described by William J. Mitchell (MIT, Smart Cities Group, http://cities.media.mit.edu/): "Our cities are fast transforming into artificial ecosystems of interconnected, interdependent intelligent digital organisms. Emerging applications in the ICT field are poised to reshape our urban environments".

In this context, wireless architectures and QoS infrastructures become nodes of a TLC network, which collects information from the surrounding areas and consequently supplies citizens with advanced infomobility services.

A very diffused and challenging problem to face is where hardware can be installed, since many areas and city centers are protected and regulated by severe rules by the Ministry of Culture and Heritage.

A possible solution is to place the whole hardware within preexistent structures, such as electricity posts, properly adapted in order to host the required technology.

A testbed based on this approach adopts "Intelligent Posts" hosting both hardware and software (Fig. 13). Such posts were installed by the WiLab group, in cooperation with Fondazione Almamater and Ghisamestieri within Villa Gandolfi Pallavicini (Bologna, Italy).

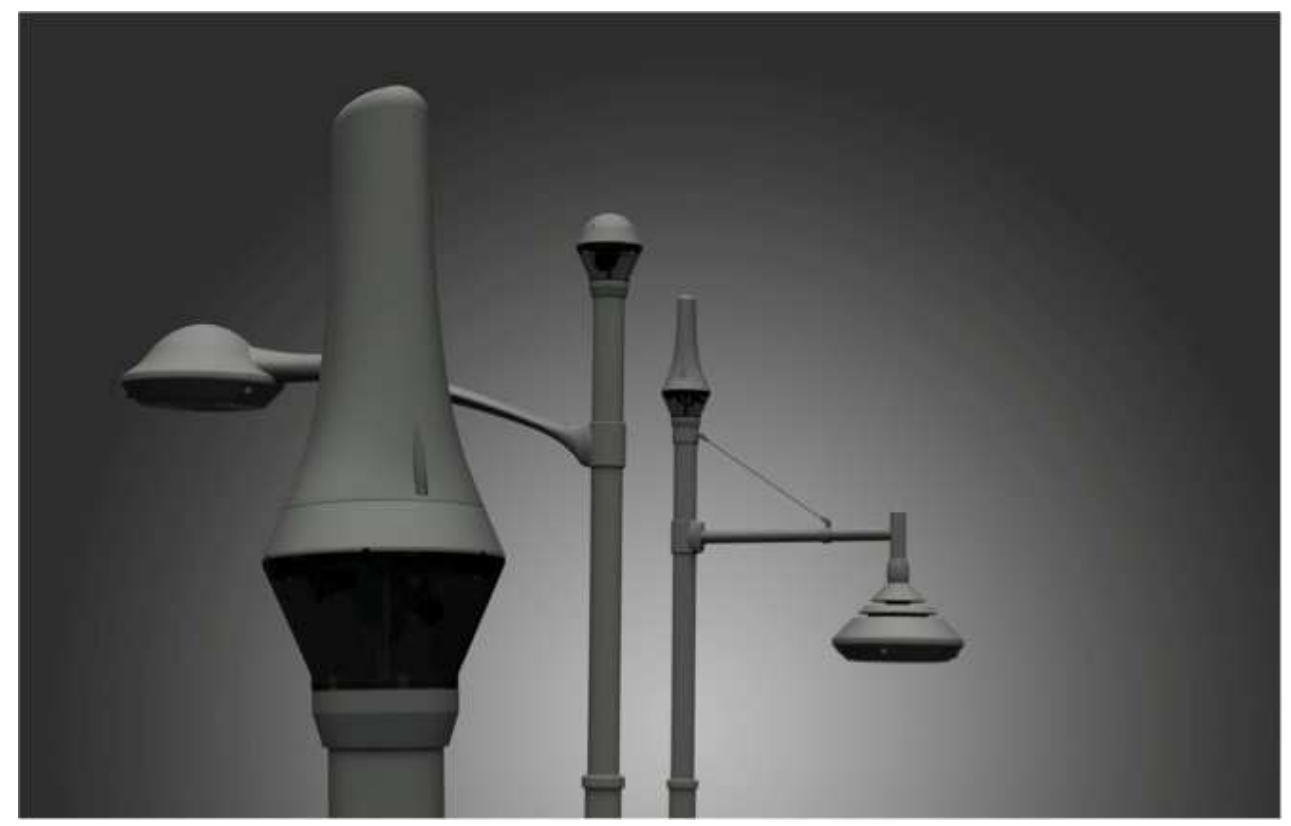

Fig. 13. Posts by Ghisamestieri for Smart Cities. 
In this case, the effective management of QoS is tested in order to supply citizens with several services, such as video surveillance (both wired and wireless data transmission involved), integrated image analysis, Internet connection within urban environments, RFID services for tourists, emergency calls, radiodiffusion, fire prevention, parking management, localization, diagnostics and control by television. In addition, sensor network data collection, traffic information, access control, mobile payments, vehicle tracking, usergenerated contents, energy management, etc.

Through QoS management, such services will be configured dynamically on the basis of bandwidth availability. According to the throughput actually available in a specific temporal slot and thanks to a constant monitoring of radio resources on each route, both services to be offered to the user and applications to be kept active can be chosen.

More specifically, the testbed is addressed to transport improvement and traffic reduction through smart navigators.

\section{Conclusions and future QoS testbed extensions}

Coming back to the tests in Sections 2, 3, PPP tunnels between the server and users can be temporarily closed, when packet transimission is slowed down by interference or machinery stops.

The first problem is that, in case the PPP LCP surveys trouble situations, the channel is closed and the client disconnected. An authomatic procedure is in charge of reconnection, but a time waste in PPP tunnel setup as well as abrupt disconnections are bound to take place.

A second problem derives from traffic limitation and control being handled by a single QoS server: in this case, data are properly limited only after they have crossed one or two links. In other words, in case an authenticated user sends an UDP data flow larger than his or her maximum upload bandwidth, such flow will be diminished only after reaching the QoS server. Meanwhile, the available bandwidth will be unproperly occupied by such flow.

On the basis of such considerations, the testbed will be extended according to two different scenarios of distributed QoS architectures [26-28]. The first one is depicted in Fig. 14 and aims at avoiding tunnel closure in case of interference and packet loss.

In case many relays occur between the client and PPPoE concentrator, packet loss can increase; the idea, thus, is to shorten the tunnel, so as to integrate the PPPoE concentrator and the transmitter. In this way, all PPP features could be maintained and its limitations diminished. The tunnel, in fact, would be established between the client's CPE and the nearest transmitter and communication between the transmitter and the main server could be based on TCP/IP.

Furthermore, if interferences between transmitter and main server would take place, packets could be relayed without PPP tunnel drops.

A disadvantage could concern uncoded communication between the main server and pylons. Possible solutions could be the activation of encrypted systems or a PPP tunnel to the main server. In this case, the user would not even perceive any link failure. 


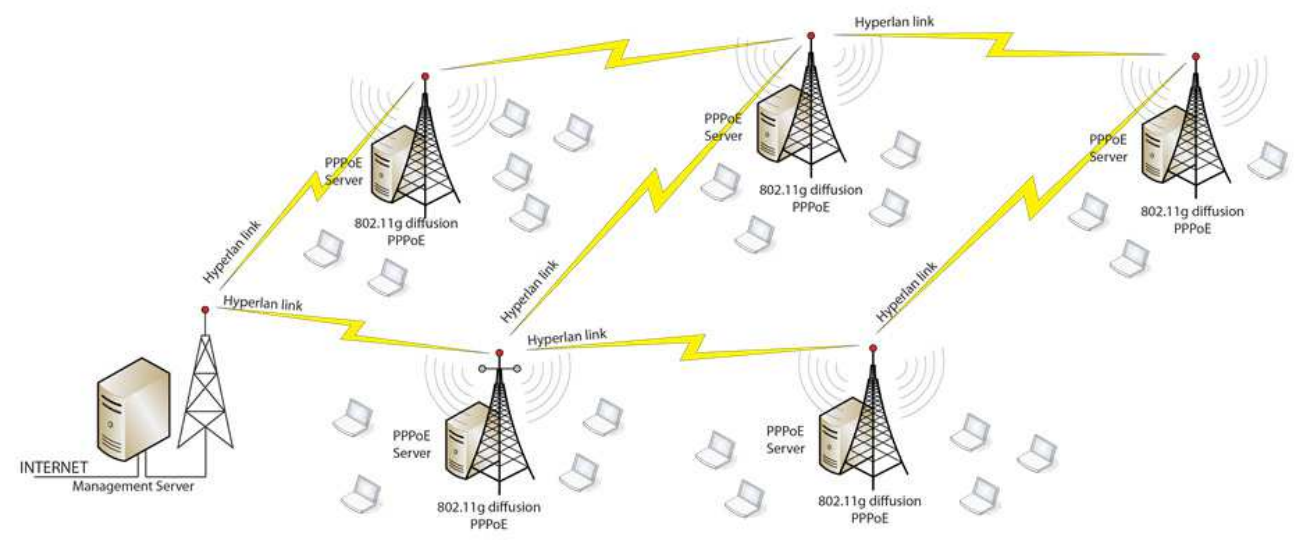

Fig. 14. first extended testbed scenario.

The second scenario (Fig. 15) aims at solving the second problem arisen: the idea is to apply the first control on users' bandwidth at the pylon.

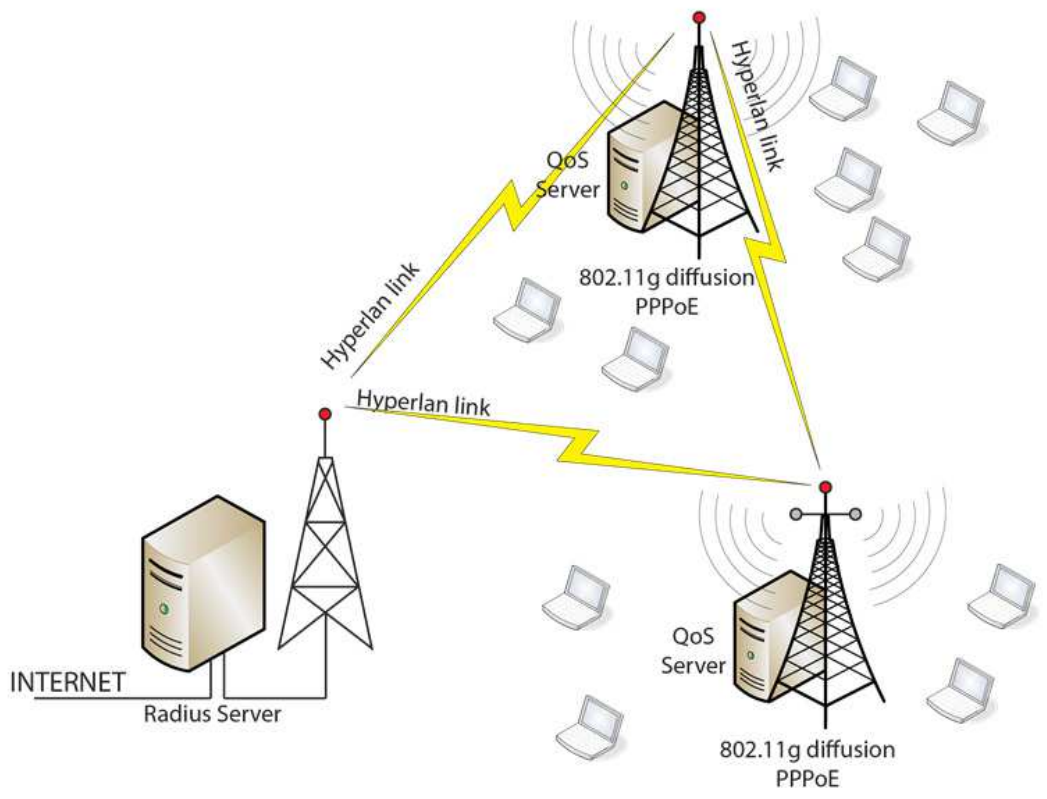

Fig. 15. second extended testbed scenario. 
As for the PPPoE concentrator, the QoS manager itself could be integrated in the transmitter. On the one hand, this kind of control logic decentralization would solve the problem of link saturation in case of heavy UDP uploads. On the other hand, the server would be spared from an exceeding traffic in case of network expansion.

Two further difficulties arise: firstly, connection plans are not anymore managed by a single server in a centralized and transparent way. In consequence, a new communication protocol is required for the authomatic configuration of devices on the pylon when the main server configuration changes.

In addition, logic decentralization can cause more frequent failures of important components. If a failure occurs of QoS or PPPoE components, thus, an infrastructure is needed that prevents connections from being denied.

A switch, for instance, could be used to disconnect out of order devices and the main server would be in charge of guaranteeing connectivity until the problem is solved.

At present, the idea is to avoid a complete implementation of the above scenarios, using simulation tools instead. In particular (Fig. 16), the QoS impact could be evaluated through the joint use of the actually developed parts and simulators.

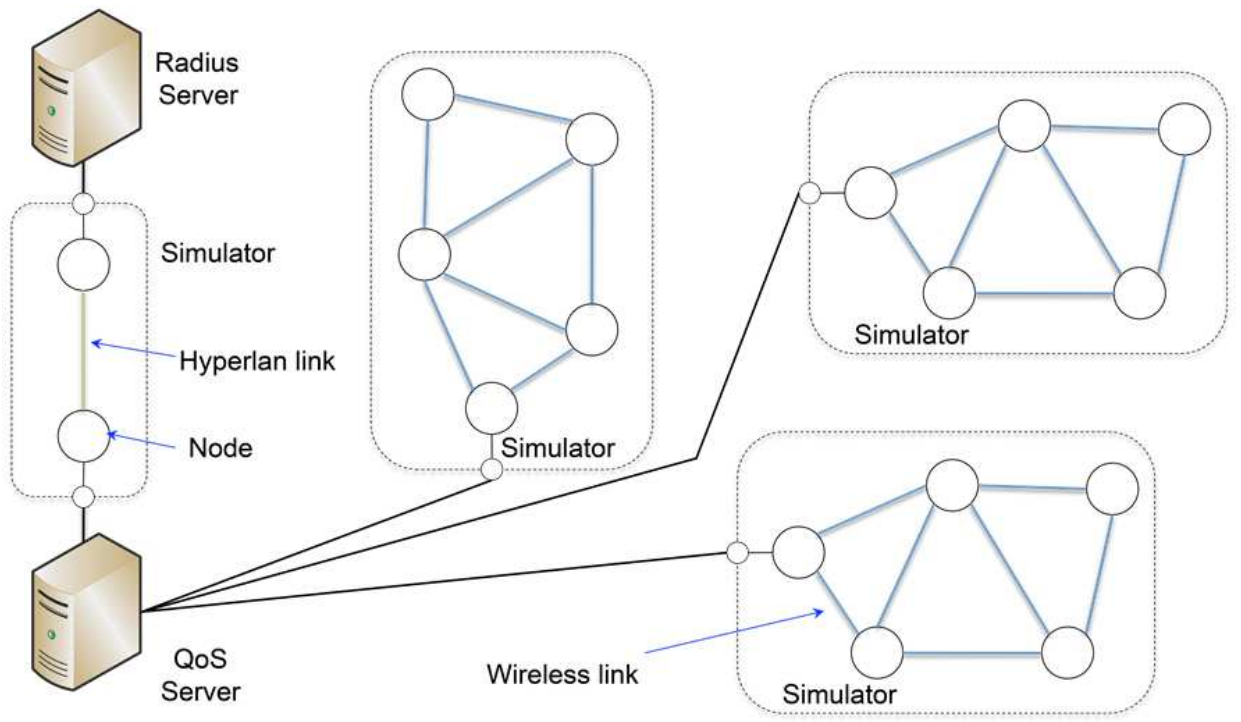

Fig. 16. A schema for the simulation of QoS server impact. 


\section{Acknowledgment}

More than an acknowledgment, a dedication: To the little Gabriele Toppan, the son of Paolo and the nephew of Andrea, go our very best wishes to grow up strong, responsible and enthusiastic about life and its numerous miracles.

\section{References}

[1] Gringeri, S.; Shuaib, K.; Egorov, R. et al.; Traffic shaping, bandwidth allocation, and quality assessment for MPEG video distribution over broadband networks, Network, IEEE, 12 , 6, pp. 94 - 107, 1998, doi: 10.1109/65.752648

[2] Frank Yong Li; Stol, N.; QoS provisioning using traffic shaping and policing in 3rdgeneration wireless networks, in Proc. of IEEE Wireless Communications and Networking Conference, 2002, 1, 139 - 143, 2002, doi: 10.1109/WCNC.2002.993478

[3] Yongdong Wang; Jurczyk, M.; Impact of traffic shaping in ATM networks on video quality, in Proc. of International Workshops on Parallel Processing, 1, 485 - 492, 2000, doi: 10.1109/ICPPW.2000.869154

[4] Gozalvez, D.; Monserrat, J.F.; Calabuig, D., et al; Policy-based channel access mechanism selection for QoS provision in IEEE 802.11e, Vehicular Technology Magazine, IEEE, 2, 3, 29-34, 2007, doi: 10.1109/MVT.2008.915326

[5] Flegkas, P.; Trimintzios, P.; Pavlou, G.; A policy-based quality of service management system for IP DiffServ networks, Network, IEEE, 16, 2, 50-56, 2002, doi: $10.1109 / 65.993223$

[6] Fangming Zhao; Lingge Jiang; Chen He; Policy-based radio resource allocation for wireless mobile networks, in Proc. of IEEE International Conference on Neural Networks and Signal Processing, 476-481, 2008, doi: 10.1109/ICNNSP.2008.4590396

[7] Conchon, E.; Pérennou, T.; Garcia, J. Et al.; W-NINE: A Two-Stage Emulation Platform for Mobile and Wireless Systems, EURASIP Journal on Wireless Communications and Networking, 2010, Article ID 149075

[8] Heithecker, S.; do Carmo Lucas, A.; Ernst, R.; A High-End Real time Digital Film Processing Reconfigurable Platform, EURASIP Journal on Embedded Systems, 2007, Article ID 85318

[9] Chang Wook Ahn; Ramakrishna, R.S.; QoS provisioning dynamic connection-admission control for multimedia wireless networks using a Hopfield neural network, Vehicular Technology, IEEE Transactions on, 53, 1, 106-117, 2004, doi:10.1109/TVT.2003.822000

[10] Xiang Chen; Bin Li; Yuguang Fang; A dynamic multiple-threshold bandwidth reservation (DMTBR) scheme for QoS provisioning in multimedia wireless networks, Wireless Communications, IEEE Transactions on, 4, 2, 583-592, 2005, doi: 10.1109/TWC.2004.843053

[11] Huan Chen; Kumar, S.; Kuo, C.J.; Dynamic call admission control scheme for QoS priority handoff in multimedia cellular systems, in Proc. of IEEE Wireless Communications and Networking Conference, 114-118, 2002, doi: 10.1109/WCNC.2002.993474 
[12] Yaya Wei; Chuang Lin; Fengyuan Ren et al; Dynamic priority handoff scheme in differentiated QoS wireless multimedia networks, in Proc. of Eighth IEEE International Symposium on Computers and Communication, 131-136, 2003, doi: 10.1109/ISCC.2003.1214112

[13] Xiaorong Li; Chuah, E.; Jo Yew Tham; Kwong Huang Goh; An optimal smooth QoS adaptation strategy for QoS differentiated scalable media streaming, in Proc. of IEEE International Conference on Multimedia and Expo, 429-432, 2008, doi: 10.1109/ICME.2008.4607463

[14] Huang, J.-H.; Li-Chun Wang; Chung-Ju Chang; Capacity and QoS for a Scalable RingBased Wireless Mesh Network, IEEE Journal on Selected Areas in Communications, 24, 11, 2070-2080, 2006, doi: 10.1109/JSAC.2006.881622

[15] Bai, B; Chen, W.; Cao, Z. et al; Uplink Cross-Layer Scheduling with Differential QoS Requirements in OFDMA Systems, EURASIP Journal on Wireless Communications and Networking, 2010, Article ID 168357

[16] Montazeri, S.; Fathy, M.; Berangi, R.; An Adaptive Fair-Distributed Scheduling Algorithm to Guarantee QoS for Both VBR and CBR Video Traffics on IEEE 802.11e WLANs, EURASIP Journal on Advances in Signal Processing, 2008, Article ID 264790

[17] Almeida, M.; Sarrô, R.; Barraca, J.P. et al; Experimental Evaluation of the Usage of Ad Hoc Networks as Stubs for Multiservice Networks, EURASIP Journal on Wireless Communications and Networking, 2007, Article ID 62967

[18] Ganesh Babu, T.V.J.; Le-Ngoc, T.; Hayes, J.F.; Performance of a priority-based dynamic capacity allocation scheme for wireless ATM systems, IEEE Journal on Selected Areas in Communications, 19, 2, 355-369, 2001, doi: 10.1109/49.914513

[19] Naser, H.; Mouftah, H.T.; A class-of-service oriented packet scheduling (COPS) algorithm for EPON-based access networks, in Proc. of 7 th Int. Conference on Transparent Optical Networks, 232-236, 2005, doi: 10.1109/ICTON.2005.1505793

[20] Song, S.; Manikopoulos, C.N.; A Priority-based Feedback Flow Control System for Bandwidth Control, in Proc. of 40th Annual Conference on Information Sciences and Systems, 1645-1652, 2006, doi: 10.1109/CISS.2006.286399

[21] Zhang, F.; Verma, P.K.; Cheng, S.; Pricing, resource allocation and quality of service in multi-class networks with competitive market model, Communications, IET, 5, 1, 51-60, doi: 10.1049/iet-com.2009.0694

[22] Kamosny, D.; Novotyny, V.; Balik, M.; Bandwidth Redistribution Algorithm for Single Source Multicast Networking, in Proc. of Int. Conference on Systems and Int. Conference on Mobile Communications and Learning Technologies, 147-156, 2006, doi: 10.1109/ICNICONSMCL.2006.62

[23] UN, World urbanization prospects: The 2007 revision population database, 2008, http://esa.un.org/unup/

[24] EU, Eu mobility and transport, 2010, http:/ / ec.europa.eu/transport/publications/statistics/

[25] ecoage, Independent ecology portal, 2003, www.ecoage.net 
[26] Won-Kyu Hong, D., Choong Seon Hong, C.; A QoS management framework for distributed multimedia systems, Int. J. Network Mgmt, 13, 115-127, 2003, doi: 10.1002/nem.465

[27] Jing Li; Yongwang Zhao; Min Liu et al; An adaptive heuristic approach for distributed QoS-based service composition, in Proc. of IEEE Symposium on Computers and Communications (ISCC), 687-694, 2010, doi: 10.1109/ISCC.2010.5546721

[28] Pattara-Atikom, W.; Krishnamurthy, P.; Banerjee, S.; Comparison of distributed fair QoS mechanisms in wireless LANs, in Proc. of IEEE Global Telecommunications Conference GLOBECOM '03. 553-557, 2003, doi: 10.1109/GLOCOM.2003.1258298 


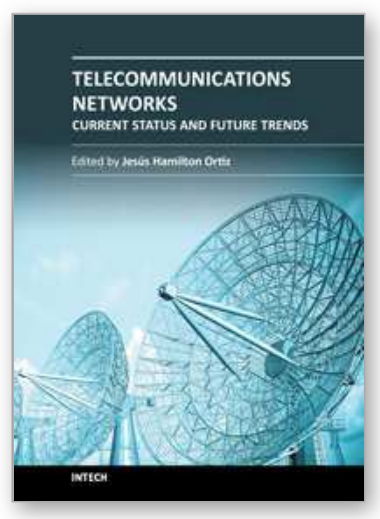

\author{
Telecommunications Networks - Current Status and Future Trends \\ Edited by Dr. Jesús Ortiz
}

ISBN 978-953-51-0341-7

Hard cover, 446 pages

Publisher InTech

Published online 30, March, 2012

Published in print edition March, 2012

This book guides readers through the basics of rapidly emerging networks to more advanced concepts and future expectations of Telecommunications Networks. It identifies and examines the most pressing research issues in Telecommunications and it contains chapters written by leading researchers, academics and industry professionals. Telecommunications Networks - Current Status and Future Trends covers surveys of recent publications that investigate key areas of interest such as: IMS, eTOM, 3G/4G, optimization problems, modeling, simulation, quality of service, etc. This book, that is suitable for both $\mathrm{PhD}$ and master students, is organized into six sections: New Generation Networks, Quality of Services, Sensor Networks, Telecommunications, Traffic Engineering and Routing.

\title{
How to reference
}

In order to correctly reference this scholarly work, feel free to copy and paste the following:

A. Toppan, P. Toppan, C. De Castro and O. Andrisano (2012). A Testbed About Priority-Based Dynamic Connection Profiles in QoS Wireless Multimedia Networks, Telecommunications Networks - Current Status and Future Trends, Dr. Jesús Ortiz (Ed.), ISBN: 978-953-51-0341-7, InTech, Available from: http://www.intechopen.com/books/telecommunications-networks-current-status-and-future-trends/a-testbedabout-priority-based-dynamic-connection-profiles-

\section{INTECH}

open science | open minds

\author{
InTech Europe \\ University Campus STeP Ri \\ Slavka Krautzeka 83/A \\ 51000 Rijeka, Croatia \\ Phone: +385 (51) 770447 \\ Fax: +385 (51) 686166 \\ www.intechopen.com
}

\author{
InTech China \\ Unit 405, Office Block, Hotel Equatorial Shanghai \\ No.65, Yan An Road (West), Shanghai, 200040, China \\ 中国上海市延安西路65号上海国际贵都大饭店办公楼 405 单元 \\ Phone: +86-21-62489820 \\ Fax: +86-21-62489821
}


(C) 2012 The Author(s). Licensee IntechOpen. This is an open access article distributed under the terms of the Creative Commons Attribution 3.0 License, which permits unrestricted use, distribution, and reproduction in any medium, provided the original work is properly cited. 